\section{Modelling the impact of process variables in community fluoridated milk schemes on a population of UK schoolchildren}

\author{
G. R. K. Foster, ${ }^{1}$ M. C. Downer ${ }^{2}$ and M. Tickle 3
}

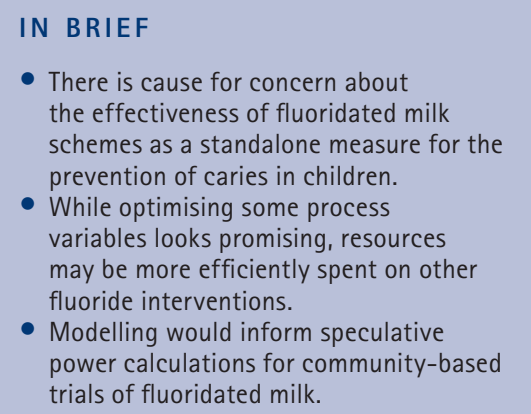
trials of fluoridated milk.

\begin{abstract}
Background Dental caries is a public health problem. Fluoridated milk (FM) schemes are used as a preventive measure. The impact of process variables in these schemes is not understood. Methods Process variable data on the number of days of consumption, attendance, volume consumed, parental consent together with the proportion of children drinking FM at 7and 11-years old were aggregated from eight schemes in the UK. The impact of process variables was modelled in an 'averaged' scheme (reduced in effectiveness by process variables) and compared with a notional 'ideal' one in which no process variables operate. Parental consent was analysed according to socio-economic groupings. Results Proportion of days per year FM was consumed: 0.52 . Values for process variables were: attendance rate 0.94 ; proportion of milk consumed 0.91; proportion of children with parental consent at 5 years 0.65 ; proportion drinking FM at 7 and 11 years respectively 0.54 and 0.27 . No clear trends were observed for parental consent across socio-economic groupings. Conclusion Modelling suggests that due to the cumulative impact of process variables, there is cause for concern about the effectiveness of FM schemes as currently managed in the UK as a standalone public health measure for the prevention of caries.
\end{abstract}

\section{INTRODUCTION}

Dental caries remains a significant public health problem. ${ }^{1}$ Water fluoridation is seen as the most effective public health measure for preventing dental caries. ${ }^{2}$ Where water fluoridation cannot be implemented, community fluoridated milk (FM) schemes may be considered. ${ }^{3}$ Since 1993, FM has been used as a prophylactic measure in 16 districts in the UK where the level of dental caries has been high and it has not been possible to introduce water fluoridation.

The benefits of FM are not fully established. ${ }^{4}$ Clinical studies on its effectiveness show great variation in the conduct of the interventions and their applicability in the UK to estimate effectiveness is difficult. ${ }^{5}$ Furthermore, the evidence from

\footnotetext{
"Manchester University Dental School, Higher Cambridge Street, Manchester, M15 6LP; ${ }^{2}$ Honorary Professor, University College London and Honorary Professor, University of Manchester/Oral Health Consultancy Services (OHCOSS), Bristol: ${ }^{3}$ Professor of Dental Public Health \& Primary Care, Oral Health Unit, National Primary Care R\&D Centre, School of Dentistry, University of Manchester

${ }^{*}$ Correspondence to: Dr G.R.K. Foste

Email:grk@MalvernFosters.me.uk
}

Online article number E17

Refereed Paper - accepted 03 March 2011

DOI: 10.1038/sj.bdj.2011.388

${ }^{\circledR}$ British Dental Journal 2011; 210: E17 studies in the UK appears contradictory. Effectiveness has been demonstrated in a cross-sectional study but not in a longitudinal study. ${ }^{6,7}$

The lack of certainty of their effectiveness makes it difficult for UK National Health Service (NHS) public health planners to advocate new FM schemes. Ideally, a public health trial to investigate the costs and benefits of a scheme is required. However, a pragmatic randomised controlled trial (RCT) would require a large number of subjects, is very expensive and would be difficult to manage because of our lack of understanding of the effects of process issues or variables on delivery of the intervention. The impact of some process variables (eg parental consent) may be affected by the socio-economic status (SES) of the target population. Future research and development of FM schemes needs to address the impact of process variables $^{7}$ and the effect of SES on process variables.

An RCT would not necessarily identify the critical points in the process of delivering an FM scheme, which may prevent the intervention from being effective. Therefore, an alternative approach for estimating the effectiveness of an FM scheme needs to be considered prior to conducting an expensive, large RCT. Modelling the impacts of the different process variables on the delivery of an FM scheme using empirical data from current schemes may provide an initial step towards estimating effectiveness, and give some indication as to whether mounting a community clinical trial would be a worthwhile undertaking. ${ }^{8}$ Modelling would also help inform power calculations when designing a trial.

This study assessed the impact of process variables on the delivery of an FM scheme as a means of estimating effectiveness in reducing caries in a population of children in the UK using a modelling approach. A secondary aim was to investigate the effect of SES on parental consent in FM schemes.

\section{METHOD}

\section{Design}

The study sample was a population of UK children in schools which participate in FM schemes. The study was chosen to use only existing anonymised shelf and audit data and not to involve direct contact with individual children. Consequently, formal review and approval was not required by the University Ethics Committee. 
Sensitivity analysis was used to model the impact of the key process variables on the delivery and effectiveness of an FM scheme and to explore what improvements might be possible. The relationship between SES and parental consent for FM was investigated in one scheme using Index of Multiple Deprivation (IMD) ${ }^{9}$ scores as a proxy measure for SES. Higher IMD values relate to higher levels of material and social deprivation.

\section{Data sources}

The key process variables with the potential to influence the effectiveness of a scheme were identified from the literature ${ }^{7}$ and through discussions with project officers involved in FM schemes. Data were collected from eight districts covering a total of 305 schools relating to over 22,600 children (in three school year groups) out of the possible 43,000 (from eight school year groups) total of children enrolled in UK FM schemes. Schemes which were experiencing problems or did not routinely collect the data required for this study were excluded. The inclusion of most of the larger schemes ensured data were available for each of the process variables of interest. Data were collected for children in year groups reception, year 2 and year 6 in each school (average ages of children were 5, 7 and 11 years respectively). These were chosen to coincide with milestones in the education process.

The following process variables were investigated (data sources in square brackets): proportion of the year (190 school days) when FM should be available $\left[{ }^{10}\right]$; loss of children from the scheme through school absence for 2006 using attendance rate as the complement of absence $\left[{ }^{11}\right]$; proportion of milk consumed daily [data source outlined below]; parental consent for FM at age 5 years and 'continuation' (the converse of loss of effectiveness over time) from age 5-7 years and from 7-11 years [calculated from audit data from UK FM schemes]; SES at individual level from children in one FM scheme using IMD (2004) scores [anonymized data from the child health database for that district].

The mean volume of school milk consumed was estimated in a sample of 15 schools in one district. Residual volumes of milk left in the carton were measured for 5- and 11-year-olds.

Table 1 Mean values and mean optimised values for process variables for an averaged FM scheme and an optimised scheme

\begin{tabular}{|l|l|l|l|l}
\hline Process variable & Mean value & $\begin{array}{l}\text { Highest } \\
\text { scheme value }\end{array}$ & $\begin{array}{l}\text { Lowest } \\
\text { scheme value }\end{array}$ & $\begin{array}{l}\text { Optimised } \\
\text { value }\end{array}$ \\
\hline $\begin{array}{l}\text { Proportion of days in year when } \\
\text { FM available }\end{array}$ & 0.52 & & 0.71 \\
\hline Attendance rate & 0.94 & & & \\
\hline $\begin{array}{l}\text { Proportion of milk in } \\
\text { carton consumed }\end{array}$ & 0.91 & & & \\
\hline Consent at 5 years & 0.65 & 0.82 & 0.53 & 0.82 \\
\hline Continuation from 5-7 years & 0.83 & & & 0.89 \\
\hline Continuation from 7-11 years & 0.49 & & & 0.62 \\
\hline Proportion drinking FM at age 7 & 0.54 & 0.73 & 0.39 & 0.73 \\
\hline Proportion drinking FM at age 11 & 0.27 & 0.46 & 0.23 & 0.46 \\
\hline
\end{tabular}

Table 2 Frequency table of number of schools in different ranges of percentage of children drinking $\mathrm{FM}$

\begin{tabular}{|c|c|c|c|}
\hline \multirow{2}{*}{$\begin{array}{l}\text { Range of percentage } x \text { of } \\
\text { children drinking FM }\end{array}$} & \multicolumn{3}{|c|}{ Number of schools in range } \\
\hline & $\begin{array}{l}\text { Drinking FM } \\
\text { at age } 5\end{array}$ & $\begin{array}{l}\text { Drinking FM } \\
\text { at age } 7\end{array}$ & $\begin{array}{l}\text { Drinking FM } \\
\text { at age } 11\end{array}$ \\
\hline$x=0$ & 5 & 4 & 7 \\
\hline $0<x \leq 5$ & 0 & 2 & 7 \\
\hline $5<x \leq 10$ & 5 & 5 & 20 \\
\hline $10<x \leq 15$ & 5 & 8 & 15 \\
\hline $15<x \leq 20$ & 3 & 8 & 14 \\
\hline $20<x \leq 25$ & 5 & 14 & 19 \\
\hline $25<x \leq 30$ & 5 & 14 & 16 \\
\hline $30<x \leq 35$ & 14 & 14 & 16 \\
\hline $35<x \leq 40$ & 12 & 16 & 16 \\
\hline $40<x \leq 45$ & 19 & 19 & 5 \\
\hline $45<x \leq 50$ & 18 & 22 & 11 \\
\hline $50<x \leq 55$ & 11 & 11 & 3 \\
\hline $55<x \leq 60$ & 21 & 21 & 5 \\
\hline $60<x \leq 65$ & 15 & 16 & 3 \\
\hline $65<x \leq 70$ & 16 & 29 & 8 \\
\hline $70<x \leq 75$ & 16 & 14 & 1 \\
\hline $75<x \leq 80$ & 18 & 17 & 2 \\
\hline $80<x \leq 85$ & 28 & 13 & 0 \\
\hline $85<x \leq 90$ & 23 & 10 & 0 \\
\hline $90<x \leq 95$ & 16 & 14 & 0 \\
\hline $95<x \leq 100$ & 37 & 10 & 2 \\
\hline Total schools & 292 & 281 & 170 \\
\hline
\end{tabular}

\section{Calculations and data analysis}

Consent is taken to be the proportion of children drinking FM at age 5 years. Continuation from age 5-7 years is the proportion drinking FM at age 7 divided by consent. Continuation from age 7-11 years is the proportion drinking FM at age 11 divided by the proportion drinking FM at age 7 .

The volumes of milk consumed daily by children were analysed using independent 
samples t-tests, with the Statistical Package for Social Sciences (SPSS for Windows 11.5).

Sensitivity analysis enables estimates of attenuation at a population level due to each process variable. The attenuation of each process variable on effectiveness was assumed to be linear. The inputs (process variables) which were amenable to improvement were varied to investigate the impact of potential improvements on effectiveness. The number of days FM is consumed was increased to show the effect of weekend consumption in term time (taken as 35 weekends), and consent at age 5 years and proportions drinking FM at 7 and 11 years were increased to model the best empirical values recorded from UK FM schemes. Optimising the observed proportions drinking FM is preferred here to optimising the actual process variables for continuation, since the latter are quotients of values which might be correlated.

The association between parental consent for FM and SES at the individual level was investigated by dividing the range of IMD values for children drinking FM and ordinary milk into quintiles. A bar chart was plotted for the proportion of these children with parental consent for FM in each quintile.

\section{RESULTS}

Table 1 summarises the mean values of process variables, and optimised values where appropriate. Confidence intervals for consent could not be determined. The value for consent is the average over the age 5 population of the included FM schemes $(8,697$ children). The frequency distribution of consent within schools (Table 2) is clearly far from any recognisable distribution. Consent appears to be strongly related to the school environment. This environment is also evident in the distributions of proportions drinking FM at ages 7 and 11 years (also in Table 2). In the absence of confidence intervals, the highest and lowest results for consent (seven schemes) are shown in Table 1. For this calculation schemes were only included if they contained relevant data for at least ten schools. The bottom two rows in Table 1 show the proportions drinking FM at ages 7 and 11, with the highest and lowest district scheme values (age 7 - six schemes, age 11 - four schemes),

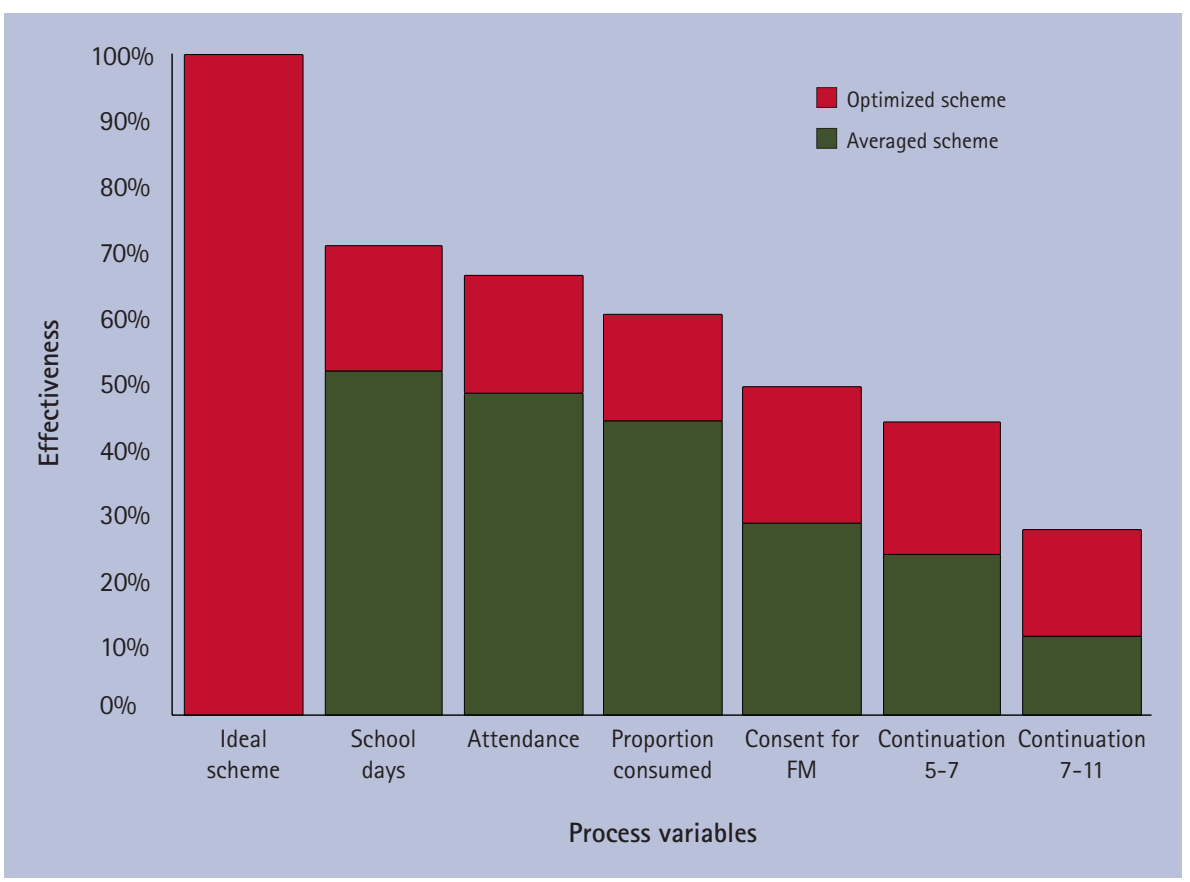

Fig. 1 Bar chart to show the attenuation due to process variables

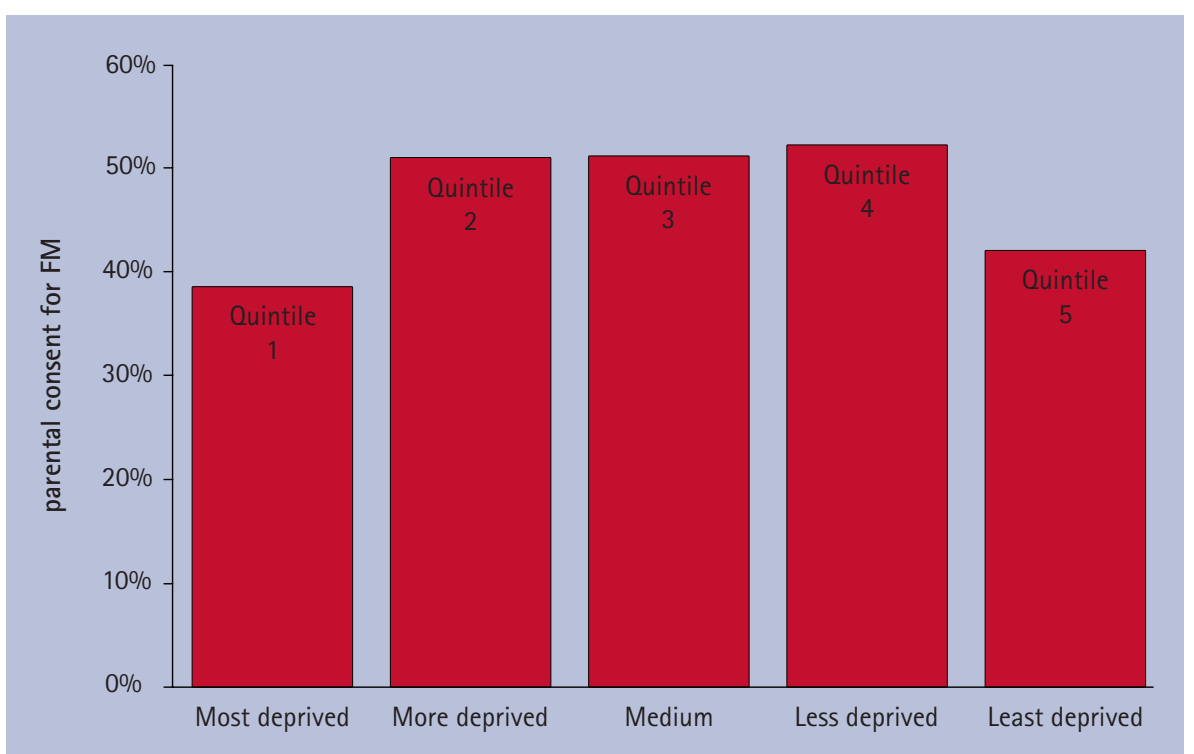

Fig. 2 Parental consent for FM by quintiles of IMD determined from the postcode of children at 5 years old in participating schools in one FM scheme

and with optimised values being the highest. The optimised values for continuation in the table are calculated from these optimised proportions together with the optimised consent.

Milk consumption data comprised 567 samples from children with a mean age of 5 years and 391 from children with mean age 11 years. There were significant differences ( $p<0.001$ ) for the volumes consumed between the 5- and 11-yearolds. The means (with 95\% CI) for volume of milk consumed were $166 \pm 4 \mathrm{ml}$ for 5-year-olds and $180 \pm 3.4 \mathrm{ml}$ for 11-yearolds. Each carton contains an average of
$189 \mathrm{ml}$ milk. Therefore the average proportion consumed by 5- and 11-year olds is $87.8 \%$ and $95.2 \%$ respectively. There is no clear way to find an average effect of this variation. A figure part way between these two proportions is likely to be within a reasonable margin of error: 91\% was taken as the percentage of milk consumed.

Averaged process variable data in Table 1 were used to construct a bar chart (Fig. 1) to show the loss of effectiveness due to each process variable when applied to an 'ideal' FM scheme (in which no process variables operate). The dark bars relate to data from the actual schemes. The height 
of each bar represents the product of that process variable with all the previous ones. The model suggests the effectiveness of an 'averaged' FM scheme (reduced in effectiveness by averaged process variables) is reduced to $24 \%$ at age 7 and $12 \%$ at age 11 compared with an ideal scheme. The effect of including term time weekends, optimising parental consent and continuation is to increase effectiveness to $44 \%$ at age 7 and 28\% at age 11 compared with an ideal scheme. Optimisation is indicated by the addition of lighter bars ('optimized scheme') in Figure 1.

Data on 12,737 children aged 5 years were used to analyse the association between parental consent and SES. This association is shown (Fig. 2) for the percentage of children with parental consent for FM at age 5 years by quintile of IMD determined from the postcode of participating children. The population was least represented from the higher socio-economic groups (quintiles 4 and 5). Children in the most deprived quintile were least likely to have consent, followed closely by children in the least deprived quintile.

\section{DISCUSSION}

\section{Main finding of this study}

The model reveals that the number of days FM is consumed, consent, and continuation from 5-7 and 7-11 years are the key process variables. The effect of these process variables substantially attenuates the optimal delivery of the scheme and this is expected to reduce the effectiveness compared with an ideal scheme. The use of optimised input values rather than averaged values suggests there may be potential for optimising (perhaps doubling) the effectiveness of the UK scheme.

Trends in parental consent for FM across socio-economic grouping were not observed.

\section{What is already known on this topic}

A recent study reported a 75\% reduction in caries increment (using ordinary milk as a control) when milk supplemented with fluoride and probiotic lactobacilli bacteria was provided to preschool children. ${ }^{12}$ The large effect size might suggest that process variables do not significantly attenuate the effectiveness of FM. Since probiotic lactobacilli might inhibit the growth of the bacteria implicated in the development of dental caries and it is not possible to differentiate the contributing effect of each variable, this study is unsuitable as a baseline for the effectiveness of FM.

A review of 15 clinical studies on the effectiveness of FM concluded that it was not generally possible to draw firm conclusions about the role of process variables on the effectiveness of FM schemes in preventing dental caries. The authors stated that it would be desirable for the number of days a child receives FM to be as many as possible. ${ }^{13}$ The effects of the other key process variables identified in this study - consent and continuation - were not considered by the authors of this review.

The relationship between consent for FM and SES is not understood. Individuals with low SES seem to benefit less from targeted preventive intervention schemes than individuals with a higher status. ${ }^{14}$ The authors of a study on a school-based caries preventive programme in Scotland found it more difficult to recruit children from the most deprived groups. ${ }^{15}$ Generally it is thought that children from a higher socioeconomic background have more favourable attitudes and oral health knowledge than children from lower socio-economic groups. ${ }^{16}$ From these findings higher levels of parental consent from higher socioeconomic groups for FM might have been expected.

\section{What this study adds}

It could be argued that since FM schemes in the UK are school-based, it is unrealistic to compare this scheme with an ideal one where there is daily exposure to fluoride. It does demonstrate the problem of limited exposure to fluoride from school day consumption of FM and the need for complementary programmes for fluoride delivery to compensate for this intermittent exposure. The modelling suggests that UK FM schemes cannot be expected to be a standalone preventive measure.

The results suggest that optimising some of the process variables in FM schemes for increased effectiveness looks promising and resources could be deployed to this end. Qualitative research has been undertaken to investigate how the effect of process variables could be optimised to improve effectiveness of FM schemes, ${ }^{17}$ if it is judged that resources would not be more efficiently spent on other interventions. FM schemes might be considered too ineffective to justify continuation. However, other fluoride programmes will also be susceptible to attenuation due to the same or additional process variables. Further, FM schemes are relatively inexpensive; ${ }^{3}$ other programmes may be more costly in terms of materials and employment of personnel. Water fluoridation, which does not require active participation and is least susceptible to attenuation from process variables, ${ }^{18}$ remains the most effective public health measure. Decision making will in the end depend on the local circumstances in terms of effective use of resources and the characteristics of the local population.

In terms of undertaking a pragmatic RCT to investigate the effectiveness of FM, a modelling approach suggests that the effect size of the intervention as it is now delivered is predicted to be very small, which would mean that a very large sample size would be required for a community trial. This makes the funding of a trial unattractive to major funding bodies due to high costs, probable operational management problems and, due to the anticipated small effect size, the impact on policy is likely to be negligible.

There is good evidence for efficacy of fluoride interventions used in schoolbased caries preventive programmes in the Cochrane systematic reviews ${ }^{19-22}$ mostly at a clinical level rather than as population interventions. The application of the modelling approach, using modelled values of identified process variables on the efficacy measure, under real-life conditions in other school-based fluoride delivery schemes needs to be considered. This needs to be backed up with empirical measurements of the effects of a newly introduced scheme.

The low consent rate for FM for children in the least deprived areas (quintile 5, Figure 2) is puzzling. Parents from higher socio-economic groups may be more aware of adverse media coverage to water fluoridation and fluoride in general. They might also question the value of FM in school, especially if they are already proactive in caring for their children's teeth at home. Whatever the reason, the unexpectedly low consent rate for quintile 5 prevents 
the expected trend of increasing parental consent rates moving from disadvantaged to affluent groups being observed. Further research is needed to investigate this phenomenon and whether it applies to other school-based public health programmes.

\section{Limitations of this study}

Additional process variables (particularly ad hoc failures in milk delivery, consumption refused because of poor quality milk, effect of drinking through a straw rather than from a cup, pupil mobility, cost of milk, and fluoride concentration in FM) were not modelled because data were not available or the variable would be difficult to quantify. Some of these factors might be expected to compromise effectiveness of the intervention further.

In practice, some children will leave the scheme at times between the chosen measurement years, and will gain some benefit from FM for a period which is not recorded. The model does not estimate this benefit.

Continuation would have been a measure of children with consent continuing to drink FM as they matured if schemes' administration and experience had been consistent over time, so that (for example) consent remained the same in successive cohorts of children. However, data for individual schools showed that there was no such consistency. Therefore, continuation includes effects of school and scheme environment (eg change of school staff or health district policy) as well as children's choice to continue drinking FM.

The modelling was based on a linear relationship for the attenuation of each process variable on effectiveness of the
FM scheme. In reality these relationships will be more complex but, in the absence of data sufficient to generate a more precise mathematical relationship, linearity is a reasonable working assumption.

\section{CONCLUSION}

Modelling the cumulative impact of process variables from FM schemes on a population of UK schoolchildren identified a major attenuation in the delivery of the scheme which will result in a reduction in effectiveness. In consequence, the modelling suggests that, under the present implementation arrangements, there is cause for concern about the effectiveness of UK FM schemes as a standalone measure for the prevention of caries.

This work was supported by the Borrow Foundation and Wirral Primary Care Trust. The authors express their thanks to P. D. Foster for mathematics support.

1. Petersen P E. World Health Organization global policy for improvement of oral health - World Health Assembly 2007. Int Dent J 2008; 58: 115-121.

2. Horowitz H S. The effectiveness of community water fluoridation in the United States. J Public Health Dent 1996; 56: 253-258.

3. Woodward S M. The implementation of community based programmes. In Bánoczy J, Petersen P E, Rugg-Gunn A J (eds) Milk fluoridation for the prevention of dental caries. Geneva: World Health Organization, 2009.

4. Yeung A, Hitchings J L, Macfarlane TV, Threlfall A, Tickle M, Glenny A M. Fluoridated milk for preventing dental caries. Cochrane Database Syst Rev 2005; 3: CD003876.

5. Mariño R. Should we use milk fluoridation? A review. Bull Pan Am Health Organ 1995; 29: 287-298.

6. Riley J C, Klause B K, Manning C J, Davies G M, Graham J, Worthington H V. Milk fluoridation: a comparison of dental health in two school communities in England. Community Dent Health 2005; 22: 141-145.

7. Ketley C E, West J L, Lennon M A. The use of school milk as a vehicle for fluoride in Knowsley, UK; an evaluation of effectiveness. Community Dent Health 2003: 20: 83-88.

8. Downer M C, Jullien J A, Speight P M. An interim determination of health gain from oral cancer and precancer screening: developing a model of population screening. Community Dent Health 1997;
14: 227-232.

9. Office of the Deputy Prime Minister. The English Indices of Deprivation 2004. http://www.communities.gov.uk/documents/communities/pdf/131206.pdf (accessed October 2010).

10. HM Government. Statutory Instrument No. 3181 The education (school day and school year) (England) regulations 1999. http://www.opsi.gov.uk/ si/si1999/19993181.htm (accessed October 2010).

11. Department for Education. Primary school (Key Stage 2) achievement and attainment tables 2006. http://www.dcsf.gov.uk/performancetables/primary_06.shtml (accessed October 2010).

12. Stecksén-Blicks C, Sjöström I, Twetman S. Effect of long-term consumption of milk supplemented with probiotic lactobacilli and fluoride on dental caries and general health in preschool children: a clusterrandomized study. Caries Res 2009; 43: 374-381.

13. Bánoczy J, Rugg-Gunn A J. Clinical studies. In Bánoczy J, Petersen P E, Rugg-Gunn A J (eds) Milk fluoridation for the prevention of dental caries. Geneva: World Health Organization, 2009.

14. Schou L, Wight C, Wohlgemuth B. Deprivation and dental health. The benefits of a child dental health campaign in relation to deprivation as estimated by the uptake of free school meals. Community Dent Health 1991; 8: 147-154.

15. Levin $K A$, Jones $C M$, Wight $C$, Valentine $C$, Topping $\mathrm{G} V \mathrm{~A}$, Naysmith R. Fluoride rinsing and dental health inequalities in 11-year-old children: an evaluation of a supervised school-based fluoride rinsing programme in Edinburgh. Community Dent Oral Epidemiol 2009; 37: 19-26.

16. Schou L, Wight C. Does dental health education affect inequalities in dental health? Community Dent Health 1994; 11: 97-100.

17. Foster G R K. The effectiveness of community milk fluoridation schemes in England. Manchester: University of Manchester, 2009. PhD thesis.

18. Griffin S O, Regnier E, Griffin P M, Huntley V. Effectiveness of fluoride in preventing caries in adults. J Dent Res 2007; 86: 410-415.

19. Marinho V C C, Higgins J P T, Logan S, Sheiham A. Fluoride gels for preventing dental caries in children and adolescents. Cochrane Database Syst Rev 2002; (1): CD002280. DOI: 10.1002/14651858.CD002280.

20. Marinho V C C. Higgins J P T, Logan S, Sheiham A. Fluoride varnishes for preventing dental caries in children and adolescents. Cochrane Database Syst Rev 2002; (1): CD002279. DOI: 10.1002/14651858. CD002279.

21. Marinho V C C, Higgins J P T, Logan S, Sheiham A. Fluoride toothpastes for preventing dental caries in children and adolescents. Cochrane Database Syst Rev 2003; (1): CD002278. DOI: 10.1002/14651858. CD002278.

22. Marinho V C C, Higgins, J P T, Sheiham A, Logan $S$. Fluoride mouth rinses for preventing dental caries in children and adolescents. Cochrane Database Syst Rev 2003; (3): CD002284. DOI: 10.1002/14651858.CD002284. 